\title{
Changes in c-Kit expression levels during the course of radiation therapy for nasopharyngeal carcinoma
}

\author{
FENG JIANG $^{1,2}$, WEI HU ${ }^{3}$, BICHENG ZHANG ${ }^{1}$, JING XU $^{1}$, YONGJIE SHUI ${ }^{1}$, \\ XIAOFENG ZHOU ${ }^{3}$, XIAOQIU REN ${ }^{3}$, XIAOZHONG CHEN $^{2}$, LI SHEN ${ }^{1,3}$ and QICHUN WEI $^{1,3}$ \\ ${ }^{1}$ Department of Radiation Oncology, The Second Affiliated Hospital, Zhejiang University School of \\ Medicine, Hangzhou, Zhejiang 310009; ${ }^{2}$ Department of Radiation Oncology, Zhejiang Cancer Hospital, \\ Hangzhou, Zhejiang 310022; ${ }^{3}$ National Ministry of Education Key Laboratory of Cancer Prevention and \\ Intervention, Zhejiang University School of Medicine, Hangzhou, Zhejiang 310009, P.R. China
}

Received May 30, 2016; Accepted August 31, 2016

DOI: 10.3892/br.2016.757

\begin{abstract}
In the era of intensity-modulated radiotherapy, distant metastasis is currently the main cause of treatment failure for nasopharyngeal carcinoma (NPC). Additional therapeutic strategies are required to control the metastasis and improve survival. One strategy is targeted therapy, for example against c-Kit. In the current study, the frequency of c-Kit expression was determined immunohistochemically in 106 NPC patients. c-Kit expression changes during the course of radiation therapy were detected in 41 cases via weekly biopsy. Twelve cases $(11.3 \%)$ had c-Kit expression scores of $3^{+}$ and $16(15.1 \%)$ had scores of $2^{+}$. Thus, c-Kit overexpression $\left(2^{+}\right.$or $\left.3^{+}\right)$was observed in $28(26.4 \%)$ patients. There were 35 $(33.0 \%)$ and $43(40.6 \%)$ patients with c-Kit expression scores of $1^{+}$and 0 , respectively. Furthermore, a trend of decreased c-Kit expression was observed after commencing radiotherapy according to the 41 NPC patients who were biopsied weekly. Therefore, c-Kit overexpression was identified to be common in NPC, and evaluating c-Kit as a therapeutic target for metastatic NPC via c-Kit overexpression subsequent to first line treatment may be of interest. To the best of our knowledge, the present study is the first to demonstrate a trend of decreased c-Kit expression during the course of radiotherapy.
\end{abstract}

\section{Introduction}

Nasopharyngeal carcinoma (NPC) has an incidence rate of 80,000 new cases per year worldwide, making up $0.7 \%$ of all cancers $(1,2)$. However, NPC has a distinctive geographic

Correspondence to: $\mathrm{Dr} \mathrm{Li}$ Shen, Department of Radiation Oncology, The Second Affiliated Hospital, Zhejiang University School of Medicine, 88 Jiefang Road, Hangzhou, Zhejiang 310009, P.R. China

E-mail: shenlizju@163.com

Key words: nasopharyngeal carcinoma, c-Kit, metastasis, targeted therapy, radiotherapy distribution, with a high prevalence in Southeast Asia, Northeast India, and North Africa, and in the Inuits of Canada and Alaska. The rates in Southeast China are particularly high (3), with the highest rate reported to be 35/100,000 in Guangdong province (4). At the time of diagnosis, $>70 \%$ of NPC patients are at stage III and stage IV (5). Furthermore, $\leq 20 \%$ of newly diagnosed NPC patients exhibit occult distant metastases (6). Radiation therapy is the standard treatment option for NPC. With the advent of intensity-modulated radiotherapy (IMRT), local-regional control has been substantially improved and distant metastasis is currently the main cause of treatment failure (7). The 5-year distant metastasis rate has been reported to be $19 \%$ for all disease stages, and $25 \%$ for the stage III-IVB subgroup $(8,9)$. Concurrent or adjuvant chemotherapy is administered to reduce the distant metastasis rate for locally advanced NPC patients. However, certain patients, particularly the elderly and those with poor performance status, cannot tolerate the toxicities. Additional therapeutic strategies are required to control the metastasis, and improve survival and the quality of life for patients with advanced NPC. One such strategy is targeted therapy.

Proto-oncogene c-Kit, also termed tyrosine-protein kinase it or CD117, is a protein encoded in humans by the KIT proto-oncogene receptor tyrosine kinase (Kit) gene (10). The c-Kit mediated signal transduction pathways appear to be involved in regulating cell differentiation and proliferation, and antibodies to c-Kit have been widely applied in immunohistochemistry to distinguish particular types of tumour, for example, malignant gastrointestinal stroma tumours (GISTs). c-Kit expression is of particular clinical interest, as it is one of the targets of the tyrosine kinase inhibitor, imatinib mesylate (termed Gleevec or Glivec). Imatinib has been shown to exert marked clinical activity in malignant GISTs containing a Kit gene mutation or c-Kit protein expression (11).

Studies concerning c-Kit expression in NPC are rare according to our search of the scientific literature from the NCBI PubMed database. To the best of our knowledge, whether c-Kit expression levels change during the course of radiation therapy has not yet been investigated. In the present study, the expression levels of c-Kit were investigated immunohistochemically in 106 NPC cases, and the changes of 
c-Kit expression during the course of radiation therapy were evaluated in 41 of the 106 cases.

\section{Materials and methods}

Patients. The present study was approved by the Institutional Review Board of Zhejiang Cancer Hospital (Hangzhou, China). Between 2007 and 2009, a total of 106 pathologically confirmed NPC patients were treated in the same medical group of Zhejiang Cancer Hospital, including 73 males and 33 females, ranging in age from 17 to 75 years (mean age, 48 years; median age, 49 years). Ninety-seven patients received platinum-based concurrent chemoradiotherapy (38 cases with nedaplatin and 59 cases with cis-platinum) and nine cases received radiotherapy alone. Nasopharyngeal endoscopy was routinely performed at the time of diagnosis, and repeated weekly during the 6-7 week course of radiation therapy. In 41 out of the 106 NPC patients, biopsies were obtained weekly, until no tumour could be seen in the nasopharyngeal endoscopy examination (i.e., a complete response; CR). In total, 2-5 biopsies were performed during the course of radiotherapy for these 41 patients according to the status of tumour regression.

The stage of disease was determined according to the 7th edition of the Union for International Cancer Control and the American Joint Committee on Cancer staging system $(12,13)$.

Clinical information was obtained from hospital records and included gender, age, pathological classification, disease stage, and concurrent chemoradiotherapy. Patient and tumour characteristics of the analysed cases are presented in Table I.

c-Kit staining. The tissue samples were fixed in $4 \%$ buffered formalin, dehydrated in graded alcohol, and fully embedded in paraffin. Subsequently, $4-\mu \mathrm{m}$ thick sections were sliced and deparaffinized in xylene at $60^{\circ} \mathrm{C}$ for $20 \mathrm{~min}$, and hydrated through graded alcohol to distilled water. Enzymatic antigen retrieval was conducted in citric acid buffer solution $(\mathrm{pH} 6)$ for $20 \mathrm{~min}$ at $98^{\circ} \mathrm{C}$. Endogenous peroxidase was blocked in $3 \% \mathrm{H}_{2} \mathrm{O}_{2}$ in phosphate-buffered saline for $10 \mathrm{~min}$. The slides were blocked in bovine serum (Sijiqing, Hangzhou, China), followed by incubation in rabbit anti-human c-Kit (cat. no. ab32363; Abcam, Cambridge, USA) diluted in 1:100 at $4^{\circ} \mathrm{C}$ overnight. After the sections were incubated in secondary antibody conjugated with AlexaFluor 488 (cat. no. A-11008; Invitrogen; Thermo Fisher Scientific, Inc., Waltham, MA, USA) for $30 \mathrm{~min}$ at room temperature, the slides were finally counterstained with hematoxylin and mounted.

c-Kit scores. The c-Kit expression was scored using the scoring criterion as reported by Bar-Sela et al (14) with modifications as follows. All the slides were evaluated by investigators who were blinded to the patients' clinical data using an Eclipse E200 microscope (Nikon Corporation, Tokyo, Japan). The staining intensities were graded according to the percentage of stained tumour cells, using a scale of $0-3^{+}$. The positive staining appeared as cytoplasmic immunoreactivity with accentuation along the cell membranes. The score was based on a scale as follows: Positive staining of $\geq 50 \%$ of the tumour cells was considered as $3+$; positive staining of $\geq 10 \%$ and $<50 \%$ of the tumour cells was considered as $2^{+} ; 2^{+}$and $3^{+}$were considered to be overexpression. Positive staining of $<10 \%$ of the tumour cells was considered as $1^{+}$; No cellular staining was considered as 0 ; and $1^{+}$and 0 were considered as low expression.

Follow-up. The patients were followed up every 3 months for the first 2 years, then every 6 months for a further 3 years and annually thereafter. Physical examination, including palpation of the neck, direct flexible fiberoptic nasopharyngoscopy, blood examination, magnetic resonance imaging scans of the nasopharynx and neck, chest radiography, and abdominal ultrasound were performed at each follow-up visit. Whole-body bone scans were obtained at least once per year.

Statistical analysis. The patients were divided into two groups; the overexpression group and low expression group, with respect to c-Kit expression. The Pearson $\chi^{2}$ test was used to compare groups regarding categorical variables, such as gender, age, tumour stage, histology classification, and concurrent chemoradiotherapy (Table I). Progression-free survival was defined as the time from treatment to the time of disease progression. Overall survival was defined as the time from treatment to date of death from any cause. Progression-free survival, overall survival and the nasopharyngeal recurrence, regional recurrence, distant metastasis were analysed using the Kaplan-Meier curves. Data were analysed using SPSS 19.0 software (IBM SPSS, Armonk, NY, USA).

\section{Results}

Expression levels of c-Kit in NPC. In the current study, among the 106 analysed NPC cases, 12 (11.3\%) had c-Kit expression scores of $3^{+}, 16$ (15.1\%) had c-Kit expression scores of $2^{+}, 35(33.0 \%)$ had c-Kit expression scores of $1^{+}$. Negative c-Kit staining was observed in 43 cases $(40.6 \%)$ of the analysed tumours. In total, c-Kit overexpression $\left(2^{+}\right.$or $\left.3^{+}\right)$was observed in $26.4 \%$ (28 of 106) of NPC cases, and low expression ( $\left(1^{+}\right.$or 0$)$ was found in $73.6 \%$ ( 78 of 106) of cases. As shown in Table I, no difference of c-Kit expression was identified among patients with different clinical staging. Examples of staining patterns for the different c-Kit expression levels are presented in Fig. 1.

Alteration of c-Kit expression during the course of radiotherapy in NPC. In the current study, weekly nasopharyngeal endoscopies were performed during the 6-7 week course of radiation therapy. In 41 out of the $106 \mathrm{NPC}$ patients tumour biopsies were also performed weekly, until no tumour could be seen (complete response; CR) during nasopharyngeal endoscopy examination. Overall, $2-5$ biopsies were made during the course of radiotherapy for these 41 patients according to the status of tumour regression. When c-Kit expression levels at different time points for the same patients were compared, a trend of decreased c-Kit expression was observed following the start of radiotherapy. For the five cases with $3^{+} \mathrm{c}-\mathrm{Kit}$ expression at diagnosis prior to radiotherapy, three exhibited reduced c-Kit expression ( $1^{+}$and 0$)$ in the second week of radiotherapy and, thereafter, one showed reduced c-Kit expression $\left(2^{+}\right)$from the fourth week, and the other case showed stable $3^{+} \mathrm{c}-\mathrm{Kit}$ expression for the first 2 weeks of radiation and the tumour had disappeared under endoscopy in the third week. For the 8 cases 
Table I. Tumour and patient characteristics $(n=106)$.

\begin{tabular}{|c|c|c|c|c|}
\hline Characteristics & Patients & c-Kit overexpression & c-Kit low expression & P-value \\
\hline Gender & & & & 0.057 \\
\hline Male & 73 & 15 & 58 & \\
\hline Female & 33 & 13 & 20 & \\
\hline Histology & 0.822 & & & \\
\hline Differentiated & 64 & 16 & 48 & \\
\hline Non-differentiated & 42 & 12 & 30 & \\
\hline T-stage & & & & 0.635 \\
\hline $\mathrm{T} 1-\mathrm{T} 2$ & 31 & 7 & 24 & \\
\hline $\mathrm{T} 3-\mathrm{T} 4$ & 75 & 21 & 54 & \\
\hline $\mathrm{N}$-stage & & & & 0.378 \\
\hline N0-N1 & 55 & 17 & 38 & \\
\hline N2-N3 & 51 & 11 & 40 & \\
\hline M-stage & & & & 1.000 \\
\hline M0 & 102 & 27 & 75 & \\
\hline M1 & 4 & 1 & 3 & \\
\hline Stage at diagnosis & & & & 1.000 \\
\hline II-III & 60 & 16 & 44 & \\
\hline IV & 46 & 12 & 34 & \\
\hline Concurrent chemoradiotherapy & & & & 0.542 \\
\hline No CCRT & 9 & 1 & 8 & \\
\hline Nedaplatin & 38 & 11 & 27 & \\
\hline Cis-platinum & 59 & 16 & 43 & \\
\hline Total & 106 & 28 & 78 & \\
\hline
\end{tabular}

The disease stage was determined according to the 7th edition of the Union for International Cancer Control and the American Joint Committee on Cancer staging system. T, primary tumour site; N, regional lymph node involvement; M, distant metastatic spread; CCRT, concurrent chemoradiotherapy.

with $2^{+} \mathrm{c}-\mathrm{Kit}$ expression at diagnosis prior to radiotherapy, all showed reduced c-Kit expression following radiotherapy (five cases exhibited reduced c-Kit expression from the second week of the course of radiotherapy, and the other three cases exhibited reduced c-Kit expression from the third week). Seven of the 10 cases $(70.0 \%)$ with $1^{+} \mathrm{c}-\mathrm{Kit}$ expression prior to radiotherapy showed negative c-Kit expression (scored as 0 ). The other three cases retained low c-Kit expression $\left(1^{+}\right)$ during the course of radiation therapy. None of the cases with c-Kit expression scored as $1^{+}$or $2^{+}$demonstrated increased c-Kit expression subsequent to radiation. For the 18 cases with negative c-Kit expression, one case showed increased c-Kit expression (scored as $1^{+}$) after radiation, the other 15 cases maintained negative expression throughout. Examples of c-Kit expression alteration during radiation therapeutic course are presented in Fig. 2.

Treatment outcome. The median follow-up time was 50 months (range, 2-75 months). Local recurrence occurred in three patients, regional recurrence occurred in three patients and distant metastases in 15 patients: Four cases to the bone, five to the liver, four to the lung, one to the bone and lung, and the other case to the bone and liver. Fifteen patients had succumbed at the time of this analysis; three from progression of residual disease, three from locoregional recurrence and 9 as a result of distant metastasis.

The results of Kaplan-Meier analysis are presented in Fig. 3. The 5-year progression-free survival rates were $83.4 \%$ for the cases with c-Kit overexpression compared with $74.7 \%$ for those with low c-Kit expression $(\mathrm{P}=0.769)$. Statistical analysis did not identify any significant differences. The overall survival rates at 5 years were 92.4 and $79.9 \%$ for the cases with c-Kit overexpression and low c-Kit expression, respectively; the difference was not statistically significant $(\mathrm{P}=0.199)$.

\section{Discussion}

In the present study, c-Kit expression levels of patients with NPC were examined, the dynamic changes of c-Kit expression during the course of radiation were evaluated, and the correlation between c-Kit expression and clinical characteristics was analysed.

The data regarding c-Kit expression in NPC are limited. In the study by Bar-Sela et al (14), 49 NPC tissue samples were analysed for c-Kit (CD117) expression. Overexpression of 
A

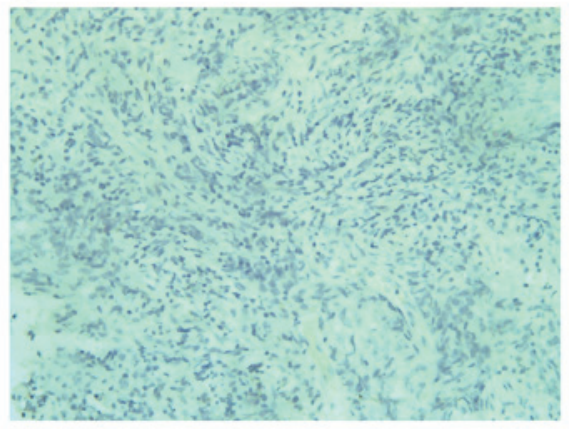

$\mathrm{C}$

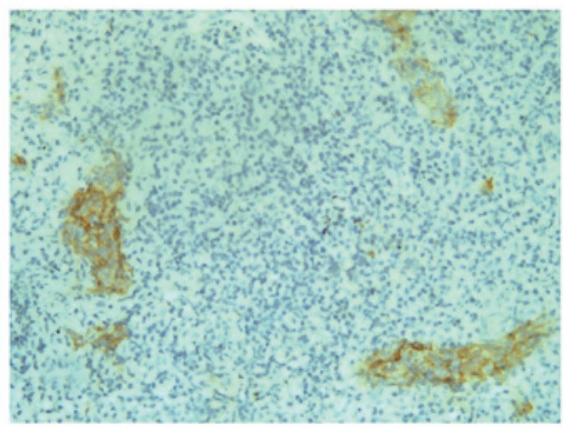

B

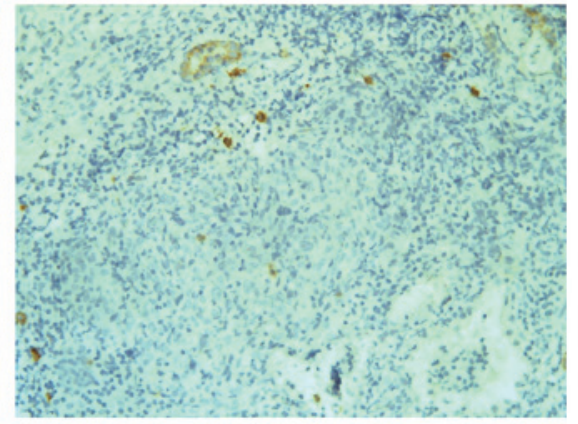

$\mathrm{D}$

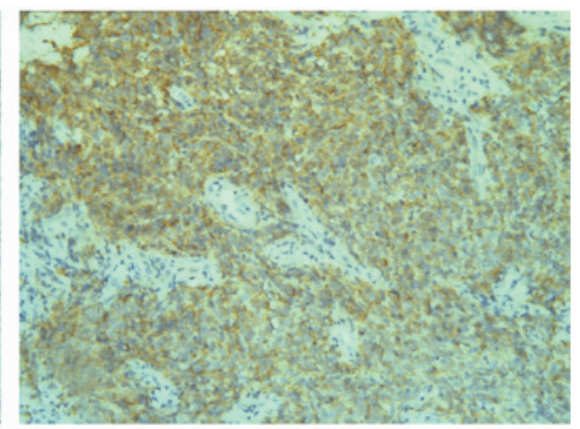

Figure 1. Immunohistochemical expression of c-Kit in nasopharyngeal carcinoma. Representative images of different c-Kit expression levels $\left(0,1^{+}, 2^{+}\right.$and $\left.3^{+}\right)$ are presented. (A) 0, (B) $1^{+}$, (C) $2^{+}$and (D) $3^{+}$. Magnification, $\mathrm{x} 20$.

A

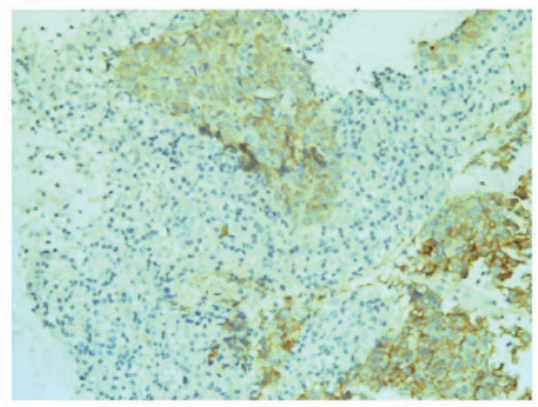

$\mathrm{C}$

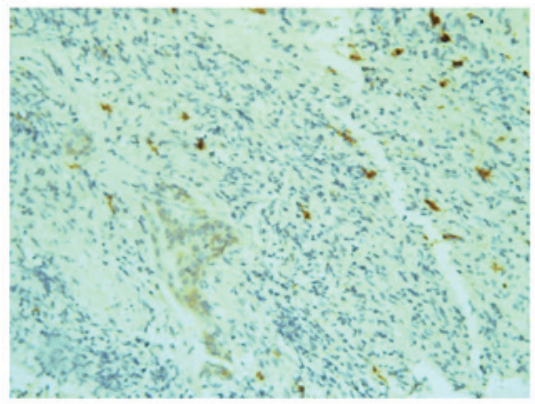

B

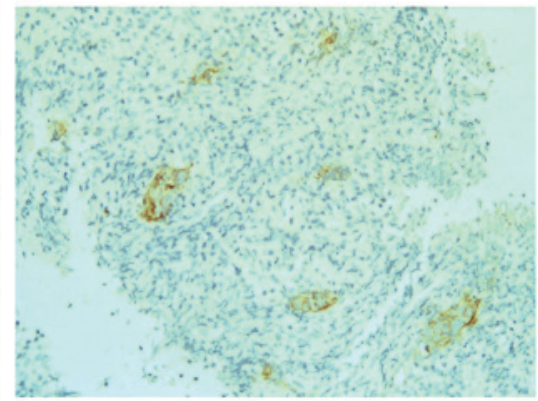

$\mathrm{D}$

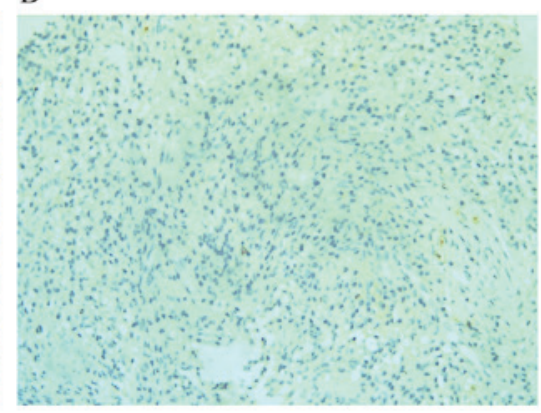

Figure 2. Weekly nasopharyngeal endoscopy and biopsy performed in one patient with nasopharyngeal carcinoma. A trend of decreased c-Kit expression was observed following radiation therapy. (A) Sample obtained prior to radiation: c-Kit staining was observed in $\geq 50 \%$ of tumour cells, and the expression scored as $3^{+}$; (B) one week after commencing radiation: positive stained tumour cells were observed in $\geq 10 \%$ and $<50 \%$ of tumour cells, c-Kit expression scored as $2^{+}$; (C) 2 weeks after commencing radiation: c-Kit staining was observed in $<10 \%$ of tumour cells, and the expression scored as $1^{+}$; (D) 3 weeks after commencing radiation: No cellular staining was observed, and c-Kit expression scored as 0. Magnification, x20.

c-Kit was identified in $33 \%(16 / 49)$ of the patients, with $18.4 \%$ (9/49) strongly positive for the c-Kit protein (staining of $>50 \%$ of the tumour cells). In another report by Bar-Sela et al (15), c-Kit overexpression was observed in $28 \%(9 / 32)$ of the adult NPC cases. In the present study, a relatively large population, of 106 NPC cases were analysed in total, and similar c-Kit scoring criteria were used as in the study Bar-Sela et al (14). Twelve $(11.3 \%)$ cases exhibited c-Kit expression that was scored as $3+$ and $16(15.1 \%)$ that was scored as $2^{+}$. Thus, c-Kit overexpression $\left(2^{+}\right.$or $\left.3^{+}\right)$was observed in $26.4 \%(28 / 106)$ 

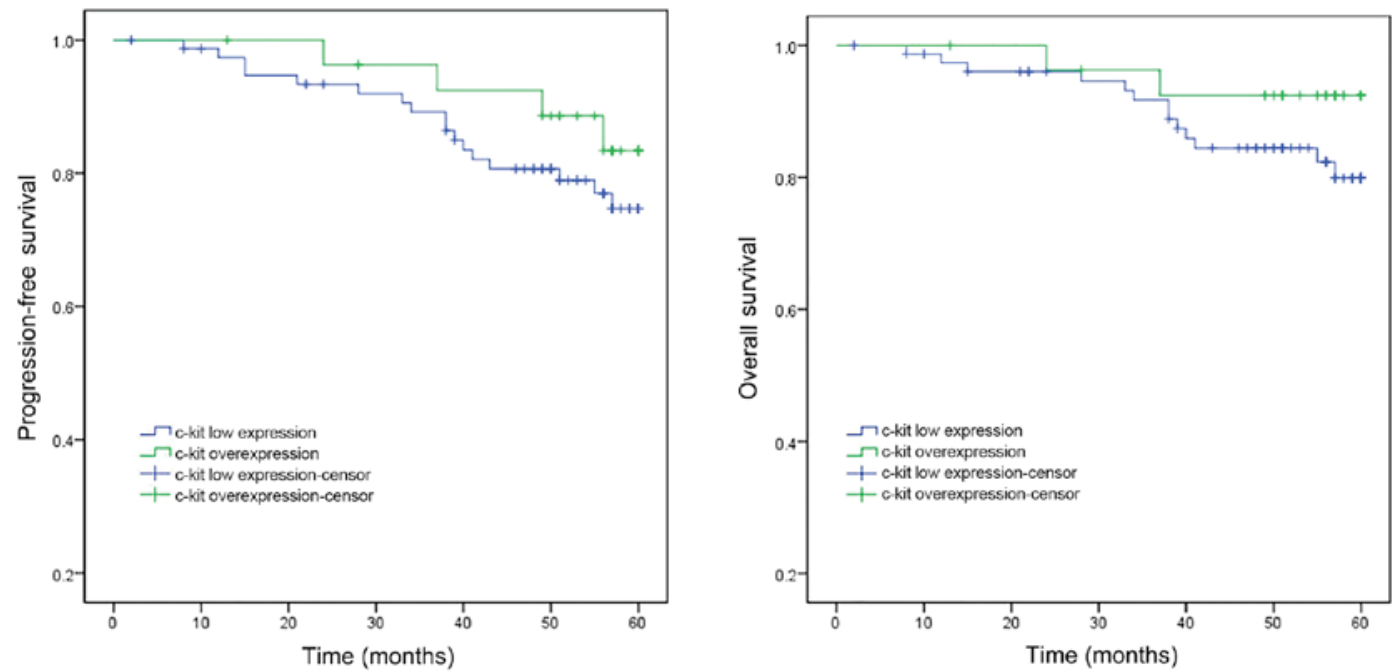

Figure 3. Kaplan-Meier curves of progression-free survival and overall survival of nasopharyngeal patients based on c-Kit staining.

of NPC cases, which is comparable to the former reports (28 and 33\%, respectively) by Bar-Sela et al $(14,15)$. However, higher expression rates of c-Kit have also been reported. In the study by Sheu et al (16), positive staining of $>1 \%$ of the tumour cells was considered to be positive for c-Kit expression, and the proportion of NPC expressing c-Kit was $86 \%$ in that series.

Children with NPC demonstrate increased c-Kit expression when compared with adults with NPC. In a study by Charfi et al (17), expression of c-kit was detected in $79 \%$ of the cases for patients aged $<30$-years-old. Bar-Sela et al $(15)$ reported on 16 NPC patients aged <20-years-old. Overexpression of c-Kit was found in $88 \%$ of the cases, as compared to $28 \%$ in adults (15). In the present study, only one patient was $<20$ years old. Furthermore, attempts were made to analyse the expression rates in different age ranges; however, no difference was identified between the younger adults and older patients.

To the best of our knowledge, the present study is the first to report whether c-Kit expression levels change during the course of radiation therapy. A trend of decreased c-Kit expression was observed subsequent to commencing radiotherapy when the c-Kit expression levels were compared at different time points for each individual patient. For the 13 cases exhibiting c-Kit overexpression $\left(2^{+}\right.$and $\left.3^{+}\right)$at diagnosis prior to radiotherapy, 12 cases demonstrated reduced c-Kit expression following radiotherapy, and the majority of patients with $1^{+} \mathrm{c}$-Kit expression showed negative staining subsequent to radiotherapy. Notably, none of the cases with c-Kit expression scored as $1^{+}$or $2^{+}$demonstrated increased $\mathrm{c}-\mathrm{Kit}$ expression following radiation. For those cases exhibiting negative c-Kit staining, the majority maintained negative expression during radiation. Although the functional involvement of c-Kit in tumorigenesis and the therapeutic response of NPC remains to be elucidated in detail, the current study provides valuable data regarding dynamic changes of c-Kit expression during the course of radiation therapy.

C-kit expression has been reported as an independent prognostic factor in patients with other types of cancer, such as small cell lung cancer (18). However, in the current study, no association was identified between $\mathrm{c}-\mathrm{Kit}$ expression and survival of NPC patients. The present results are consistent with previous reports by Bar-Sela et al $(14,15)$. In the present study, the majority of the cases were adults, with only one patient aged 17-years-old. For paediatric patients, tumours with strongly positive c-Kit expression were reported to have a lower recurrence rate (15). However, larger studies are required to evaluate c-Kit as a potential prognostic factor.

c-Kit overexpression had been reported in various NPC cell lines (19). In preclinical studies, imatinib and sunitinib induced a dose-dependent inhibitory effect on the proliferation of NPC cells $(19,20)$. As yet, to the best of our knowledge, targeted therapy against c-Kit has not been investigated in NPC patients. As c-Kit overexpression has been reported in $~ 30 \%$ of adult NPC patients $(14,15)$, it may be of interest to evaluate c-Kit as a therapeutic target for metastatic NPC patients with c-Kit overexpression, particularly for those whose first line treatment failed.

c-Kit overexpression was identified to be common in NPC, therefore, it may be of interest to evaluate c-Kit as a therapeutic target for metastatic NPC with c-Kit overexpression as a second line treatment. A trend of decreased c-Kit expression was observed during the course of radiotherapy. However, the prognostic value of c-Kit in patients with NPC remains to be elucidated.

\section{Acknowledgements}

The present study was supported by a grant from the National Natural Science Foundation of China (grant no. 81572952).

\section{References}

1. Chang ET and Adami HO: The enigmatic epidemiology of nasopharyngeal carcinoma. Cancer Epidemiol Biomarkers Prev 15: 1765-1777, 2006.

2. Jemal A, Bray F, Center MM, Ferlay J, Ward E and Forman D: Global cancer statistics. CA Cancer J Clin 61: 69-90, 2011.

3. Wang JB, Jiang Y, Liang H, Li P, Xiao HJ, Ji J, Xiang W, Shi JF, Fan YG, Li L, et al: Attributable causes of cancer in China. Ann Oncol 23: 2983-2989, 2012.

4. Jia WH, Huang QH, Liao J, Ye W, Shugart YY, Liu Q, Chen LZ, Li YH, Lin X, Wen FL, et al: Trends in incidence and mortality of nasopharyngeal carcinoma over a 20-25 year period (1978/1983-2002) in Sihui and Cangwu counties in southern China. BMC Cancer 6: 178, 2006. 
5. Yi JL, Gao L, Huang XD, Li SY, Luo JW, Cai WM, Xiao JP and Xu GZ: Nasopharyngeal carcinoma treated by radical radiotherapy alone: Ten-year experience of a single institution. Int J Radiat Oncol Biol Phys 65: 161-168, 2006.

6. Ong YK, Heng DM, Chung B, Leong SS, Wee J, Fong KW, Tan T and Tan EH: Design of a prognostic index score for metastatic nasopharyngeal carcinoma. Eur J Cancer 39: 1535-1541, 2003.

7. Lai SZ, Li WF, Chen L, Luo W, Chen YY, Liu LZ, Sun Y, Lin AH, Liu MZ and Ma J: How does intensity-modulated radiotherapy versus conventional two-dimensional radiotherapy influence the treatment results in nasopharyngeal carcinoma patients? Int J Radiat Oncol Biol Phys 80: 661-668, 2011.

8. Lee AW, Sze WM, Au JS, Leung SF, Leung TW, Chua DT, Zee BC, Law SC, Teo PM, Tung SY, et al: Treatment results for nasopharyngeal carcinoma in the modern era: The Hong Kong experience. Int J Radiat Oncol Biol Phys 61: 1107-1116, 2005.

9. Hui EP, Leung SF, Au JS, Zee B, Tung S, Chua D, Sze WM Law CK, Leung TW and Chan AT: Lung metastasis alone in nasopharyngeal carcinoma: A relatively favorable prognostic group. A study by the Hong Kong Nasopharyngeal Carcinoma Study Group. Cancer 101: 300-306, 2004.

10. Yarden Y, Kuang WJ, Yang-Feng T, Coussens L, Munemitsu S, Dull TJ, Chen E, Schlessinger J, Francke U and Ullrich A: Human proto-oncogene c-kit: A new cell surface receptor tyrosine kinase for an unidentified ligand. EMBO J 6: 3341-3351, 1987.

11. Dagher R, Cohen M, Williams G, Rothmann M, Gobburu J, Robbie G, Rahman A, Chen G, Staten A, Griebel D, et al: Approval summary: Imatinib mesylate in the treatment of metastatic and/or unresectable malignant gastrointestinal stromal tumors. Clin Cancer Res 8: 3034-3038, 2002.

12. Sobin LH, Gospodarowicz MK and Wittekind C (eds). TNM classification of malignant tumours. 7th edition. Wiley Blackwell, Chichester, 2010.

13. Edge SB, Byrd DR, Compton CC, Fritz AG, Greene FL and Trotti A III (eds). American Joint Committee on Cancer (AJCC). Cancer staging manual. 7th edition. Springer, New York, 2010.
14. Bar-Sela G, Kuten A, Ben-Eliezer S, Gov-Ari E and Ben-Izhak O: Expression of HER2 and C-KIT in nasopharyngeal carcinoma: Implications for a new therapeutic approach. Mod Pathol 16: 1035-1040, 2003

15. Bar-Sela G, Ben Arush MW, Sabo E, Kuten A, Minkov I and Ben-Izhak O: Pediatric nasopharyngeal carcinoma: Better prognosis and increased c-Kit expression as compared to adults. Pediatr Blood Cancer 45: 291-297, 2005.

16. Sheu LF, Lee WC, Lee HS, Kao WY and Chen A: Co-expression of c-kit and stem cell factor in primary and metastatic nasopharyngeal carcinomas and nasopharyngeal epithelium. J Pathol 207: 216-223, 2005.

17. Charfi S, Khabir A, Ayadi L, Mseddi M, Makni H, Gorbel A, Daoud J, Frikha M, Jlidi R, Busson P, et al: Expression of c-kit in North African nasopharyngeal carcinomas: Correlation with age and LMP1. Cancer Radiother 11: 247-251, 2007 (In French).

18. Rohr UP, Rehfeld N, Pflugfelder L, Geddert H, Müller W, Steidl U, Fenk R, Gräf T, Schott M, Thiele KP, et al: Expression of the tyrosine kinase c-kit is an independent prognostic factor in patients with small cell lung cancer. Int J Cancer 111: 259-263, 2004.

19. Huang PY, Hong MH, Zhang X, Mai HQ, Luo DH and Zhang L: C-KIT overexpression and mutation in nasopharyngeal carcinoma cell lines and reactivity of Imatinib on these cell lines. Chin J Cancer 29: 131-135, 2010.

20. Hui EP, Lui VW, Wong CS, Ma BB, Lau CP, Cheung CS, Ho K, Cheng $\mathrm{SH}, \mathrm{Ng} \mathrm{MH}$ and Chan AT: Preclinical evaluation of sunitinib as single agent or in combination with chemotherapy in nasopharyngeal carcinoma. Invest New Drugs 29: 1123-1131, 2011. 